\title{
Emerging roles for specific fatty acids in developmental processes
}

\author{
Tracy L. Vrablik and Jennifer L. Watts ${ }^{1}$ \\ School of Molecular Biosciences, Center for Reproductive Biology, Washington State University, Pullman, Washington 99164, \\ USA
}

Animals synthesize a vast range of fatty acids serving diverse cellular functions. The roles of specific fatty acids in early development are just beginning to be characterized. In the March 15, 2012, issue of Genes \& Development, Kniazeva and colleagues (pp. 554-566) describe how the particular combination of a branched chain fatty acid and an acyl-CoA synthetase is required for critical cellular processes during early embryogenesis in Caenorhabditis elegans.

Lipids serve three vitally important roles in biological systems: energy storage, structural components of membranes, and signaling molecules. Eukaryotic cells are composed of thousands of distinct lipid species (Shevchenko and Simons 2010). Approximately 5\% of genes encode proteins that function in lipid metabolism, and synthesis of such a vast array of lipid species uses considerable cellular energy. Lipidomic initiatives, such as LIPID MAPS (Fahy et al. 2011), are devoted to the identification and characterization of these lipids, but our understanding of the functional significance of each distinct lipid is in its infancy.

\section{Structural and functional diversity of membrane lipids \\ Lipid classes are not distributed equally throughout cellu- lar membranes. The major glycerolipids and sphingolipids are distributed asymmetrically in plasma membranes, with some classes present only in the inner leaflet, and some present only in the outer leaflet. The structure of the polar head groups determines their cellular geometry; for example, "cone-shaped" lipids, such as phosphatidyletha- nolamine, contribute to membrane curvature and vesicle formation, while "cylindrical" lipids, such as phosphati- dylcholine, spontaneously form bilayers (van Meer et al. 2008). Specialized lipids perform specific functions. For example, mitochondria contain lipids in common with bacteria, such as cardiolipin, which is required for efficient oxidative phosphorylation. The plasma membrane con-}

[Keywords: BCFA; branched chain fatty acid; acyl-CoA synthetase; somatic gonad; exocytosis; inositol trisphosphate]

${ }^{1}$ Corresponding author.

E-mail jwatts@wsu.edu.

Article is online at http://www.genesdev.org/cgi/doi/10.1101/gad.190777.112. tains substantial levels of sphingolipids and cholesterol, which pack more tightly into membranes and provide stability and resistance to mechanical stress. Sphingolipids and cholesterol are key components of lipid microdomains, also known as lipid rafts. Lipid rafts coalesce by specific lipid-lipid and protein-lipid interactions to provide the optimum membrane environment for cellular signaling events as well as promote membrane bending and vesicle formation (Simons and Sampaio 2011).

In contrast to the distinct structural functions attributed to various polar head groups, less is known about the functional roles of specific fatty acids. The wide variety of fatty acid species is responsible for much of the lipid diversity in membrane and storage lipids. Natural fatty acids are typically composed of an even number of carbon atoms, the most common ranging from 10 to 24 carbons in length. They can be saturated, with all carbon-bonding positions containing hydrogens, or unsaturated, indicating the presence of one to six cis double bonds. These double bonds are inserted into fatty acid chains by fatty acid desaturases, which catalyze a mixed function oxidation reaction to reduce molecular oxygen to water, resulting in the removal of two hydrogen atoms from the fatty acid chain (Wallis et al. 2002). The presence of even one double bond greatly reduces the ability of fatty acids to pack together in membranes, allowing for increased fluidity of membranes. However, the regulation of membrane fluidity alone does not require the large repertoire of fatty acids that exists in nature.

One well-recognized function of long chain polyunsaturated fatty acids is their role as precursors for signaling molecules. Phospholipases cleave C20 and C22 polyunsaturated fatty acids from the membrane, which can then be acted on by cyclooxygenase and lipoxygenase enzymes to produce powerful short-lived, short-range hormones called prostaglandins, thromboxanes, and leukotrienes. These molecules, collectively known as eicosanoids, modulate blood pressure, reproduction, inflammation, pain, and immune responses (Haeggstrom and Funk 2011; Smith et al. 2011).

Regulating fatty acid synthesis is critical for development

A vastly underexplored field is the study of specific lipids required for development. Oocytes are typically a lipid- 
rich environment, providing energy stores and precursors for growth and membrane biosynthesis. However, emerging evidence suggests that diverse fatty acids promote development through means beyond meeting the energy requirements for growth. Defects in lipid synthesis are often associated with developmental abnormalities or lethality, but the underlying mechanisms are only beginning to be characterized.

Biosynthesis of fatty acids begins with acetyl-CoA carboxylase (ACC), which catalyzes the ATP-dependent carboxylation of acetyl-CoA to form malonyl-CoA. Malonyl-CoA is then used as the $\mathrm{C}_{2}$ unit donor for de novo fatty acid synthesis by fatty acid synthase (FAS) (Fig. 1). Mammals have two different ACC isoforms, ACC1 and ACC2, which have distinct expression patterns.

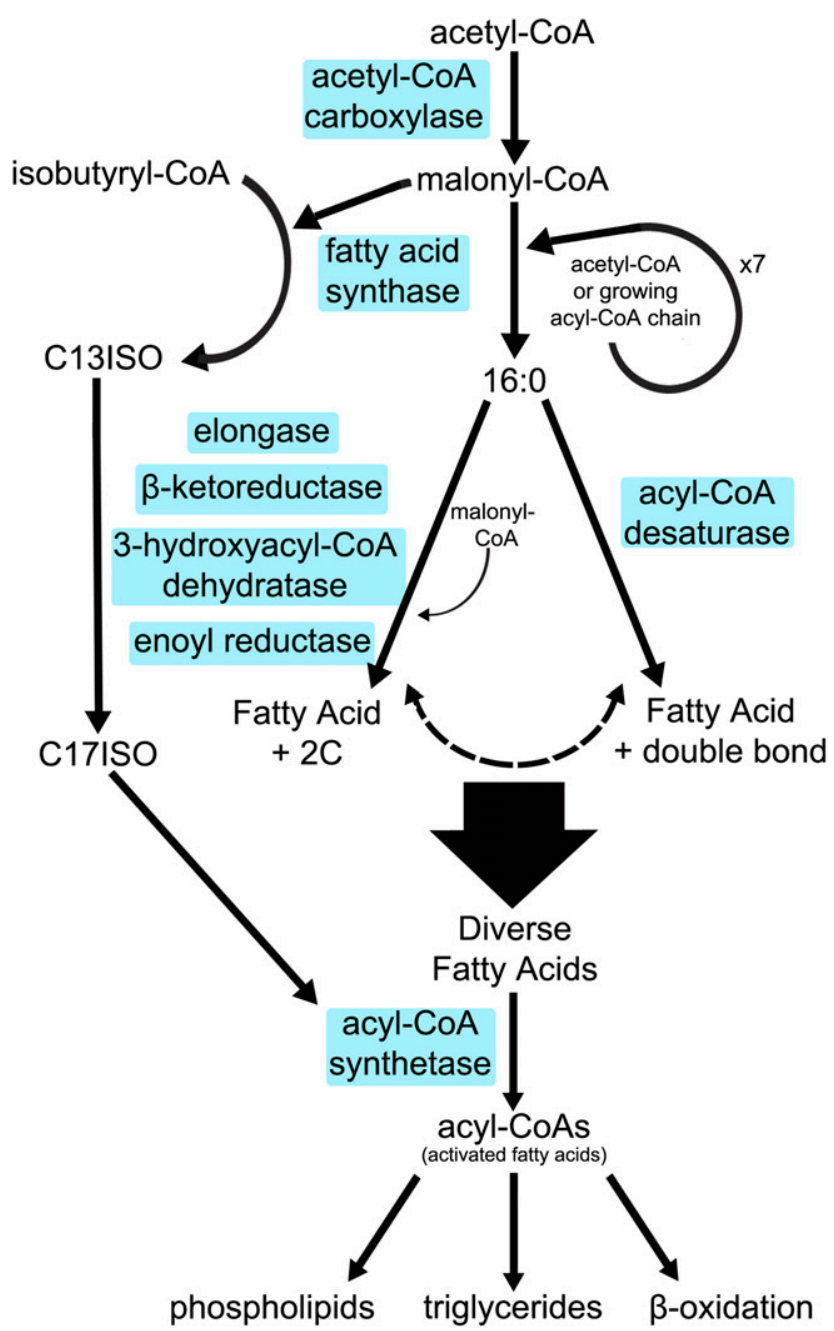

Figure 1. Overview of de novo fatty acid synthesis pathways in animals. Enzyme names are highlighted in blue boxes. Synthesis of branched chain fatty acids begins with isobutyryl-CoA, and synthesis of straight chain fatty acids begins with acetyl-CoA. Both straight and branched chain fatty acids are extended via the sequential addition of malonyl-CoA units by FAS. Straight chain fatty acids can then be elongated and/or desaturated. The fatty acid products of de novo synthesis are activated by ACS, from which they can then be incorporated into more complex lipids.
$A c c 1^{-/-}$mice are embryonic-lethal by embryonic day 8.5 (E8.5), while $A c c 2^{-/-}$mice develop normally and have increased resistance to obesity and diabetes (Abu-Elheiga et al. 2005). FAS is a multifunctional protein consisting of seven catalytic domains that builds the fatty acid chains through successive condensation reactions with malonylCoA to yield palmitoyl-CoA (16:0). FAS is expressed in nearly every tissue in humans. Fasn ${ }^{-1-}$ mice were embryonic-lethal at the preimplantation stage, and $\sim 70 \%$ of $\mathrm{Fasn}^{+/-}$mice were embryonic-lethal, indicating partial haploinsufficiency (Chirala et al. 2003). These studies highlight the importance of de novo synthesis of saturated fatty acids for development.

The palmitate end product generated by de novo synthesis through FAS is then elongated and desaturated to generate a variety of straight chain fatty acids. Elongation, like de novo fatty acid synthesis, adds $C_{2}$ units donated by malonyl-CoA to a fatty acyl-CoA. Elongation is achieved with four separate enzymatic reactions: ELOVL enzymes catalyze the condensation between the fatty acylCoA and malonyl-CoA to yield 3-ketoacyl-CoA, $\beta$-ketoreductases catalyze the reduction to 3-hydroxyacyl-CoA, 3-hydroxyacyl-CoA dehydratases catalyze the dehydration to trans-2-enoyl-CoA, and finally, enoyl reductases form the elongated acyl-CoA (Fig. 1). The ELOVL enzymes are the rate-controlling enzymes in elongation. There are currently seven known ELOVL proteins in mammals, which exhibit substrate preference and different in vivo expression patterns. Elov $14^{-/}$mice die soon after birth due to dehydration resulting from reduced levels of specific ceramides in the skin. In humans, two forms of macular dystrophy are associated with a 5-basepair (bp) deletion in Elov14. Elov15 ${ }^{-1-}$ female mice have decreased fertility, but no infertility is observed in the males (Guillou et al. 2010). Acyl-CoA desaturases introduce a double bond at a specific position on the acyl chain of long chain fatty acids (Fig. 1). Mammals have $\Delta 9, \Delta 6$, and $\Delta 5$ desaturase activities, where the $\Delta$ number indicates the position where the double bond is introduced, counting from the methyl end. Stearoyl Co-A desaturases (SCDs) possess $\Delta 9$ desaturase activity. In mice, there are four SCD isoforms, while in humans, only two isoforms have been identified so far. $S c d-1^{-/-}$mice exhibit sebaceous gland hypoplasia and alopecia, and $S c d-2^{-/-}$mice die within a day of birth. The $\Delta 5$ and $\Delta 6$ desaturase activity in mammals is encoded by fatty acid dehydrogenase 1 (Fads1) and Fads2, respectively. Fads $2^{-1-}$ mice lacking $\Delta 6$ desaturase activity have normal viability and life span, but are sterile (Guillou et al. 2010).

Acyl-CoA synthetases (ACSs) catalyze the esterification of fatty acids with coenzyme A to make activated acyl-CoA intermediates that can be elongated or incorporated into complex lipids (Fig. 1). There are 26 ACS genes in mammals, and the different ACS isoforms are hypothesized to channel fatty acids into specific downstream pathways (Ellis et al. 2010). Many of these ACS genes have been characterized for their effects on fatty acid composition in different tissues in mammals; however, the potential effects of these changes in lipid composition on development remain unclear. 
Elucidation of the roles for specific lipid species in development is complicated by gene redundancy as well as the possibility that essential lipid synthesis genes can affect multiple aspects of development. Simple metazoan models such as Caenorhabditis elegans and Drosophila melanogaster offer genetically and biochemically tractable systems in which to study lipid metabolism. The combination of forward and reverse genetics, lipid analysis, and tissue-specific expression studies has enabled dissection of lipid metabolism and regulation in the context of a whole organism. Work in these systems has revealed critical regulators of lipid metabolism, such as the insulin/insulin-like growth factor signaling pathway; however, much of this analysis has focused on lipid metabolism in the context of adiposity and metabolic syndrome (Baker and Thummel 2007; Watts 2009). A new study from the Han laboratory (Kniazeva et al. 2012) uses a combination of techniques to reveal a role for a branched chain fatty acid in the regulation of early embryogenesis in C. elegans. We first summarize recent studies in Drosophila and C. elegans revealing the importance of fatty acid synthesis and regulation of membrane composition on development.

\section{Lipid synthesis in gametogenesis and fertilization in Drosophila and C. elegans}

Lipid synthesis genes play a critical role in Drosophila gametogenesis. In Drosophila, loss of the elongase bond, homologous to human Elovl4 and Elov17, has no overt effects on morphology or viability but causes both male and female sterility. Elovl proteins elongate acyl chains longer than 18 carbon atoms into very long chain fatty acids, which are commonly found in membrane sphingolipids. Characterization of bond males revealed that spermatogenesis fails due to impaired cytokinesis during meiosis. Spermatocytes in bond males are able to form the actin-myosin contractile ring at the proper site of furrow ingression, but then the contractile ring fails to contract or contracts very slowly. Late in cytokinesis, the stalled contractile ring detaches from the plasma membrane and rapidly condenses on itself (Szafer-Glusman et al. 2008). This suggests that a specific elongase is employed to regulate the membrane composition of spermatocytes, which is important to maintain attachment of the contractile ring to withstand the stresses induced during cytokinesis.

Loss of the Drosophila homolog of Elovl6, neighbor of $a b l$ (noa), which elongates 12-16 carbon fatty acid chains, results in embryonic lethality. However, mutant strains carrying weaker alleles are viable but have defective spermatogenesis. During spermatid differentiation, an actin-rich individualization complex made up of 64 actin cones forms and transverses the sperm cyst cells, stripping the spermatids of their excess mass. Knockdown of noa expression specifically in the somatic sperm cyst cells causes the individualization complex to dissociate and the individualization cones to appear morphologically abnormal (Jung et al. 2007). Thus, Elovl6 modulation of the fatty acid composition is broadly necessary for viability but also has a specific role in maintaining the structural cytoskeletal components necessary for sperm differentiation.

Lysophospholipid acyltransferases (LPLATs) catalyze the addition of fatty acyl chains to lysophospholipids to generate phospholipids. As such, LPLATs are important regulators of membrane remodeling and composition. In Drosophila, the LPLAT transcripts oysgedart (oys) and nessy (nes) are maternally deposited in embryos, and zygotic loss of both oys and nes causes embryonic lethality in more than half of animals. Males that reach adulthood are sterile because of a failure to complete sperm individualization. The individualization complexes and actin cones appear to form properly in the oys;nes mutants, but these complexes dissociate prior to completion of sperm individualization. The Oys and Nes LPLATs preferentially act on unsaturated fatty acids, so loss of their activity leads to an increase in the proportion of phospholipids with two saturated acyl chains in both whole animals and the testes (Steinhauer et al. 2009). Thus, the maintenance of membrane phospholipid composition by LPLATs has broad roles for viability, but also a specific role in the stabilization of individualization complexes with spermatids during sperm differentiation. These studies on Elovl6 and LPLATs in Drosophila sperm differentiation highlight how different lipid synthesis genes, which regulate levels of distinct lipid species, cooperate to optimize membrane composition and function in the same developmental process.

A recent study reported a role for the synthesis of cardiolipin for efficient germ cell proliferation in C. elegans. Cardiolipin synthase catalyzes the formation of cardiolipin from phosphatidylglyerol and diacylglycerol. A strain carrying a mutation in crls-1, the homolog of cardiolipin synthases, produced a very small brood compared with wild type. Further studies revealed defects in germ cell proliferation in the crls-1 mutants, even though somatic cells divided normally in the same animals (Sakamoto et al. 2012). This suggests that the contribution of cardiolipin to mitochondrial function varies among cell types and indicates that proper membrane lipid composition in mitochondria is vital for the optimal proliferation of germ cells.

In addition to endogenous lipid synthesis, dietary intake of fatty acids can also affect lipid composition and have developmental consequences. In C. elegans, dietary supplementation of a specific polyunsaturated fatty acid, dihomo- $\gamma$-linolenic acid (DGLA), causes sterility by inducing both mitotic and meiotic germ cells to undergo cell death. Interestingly, loss of omega- 3 and $\Delta 5$ desaturase activities in fat-1;fat-4 double mutants causes accumulation of high levels of DGLA, but these worms maintain their fertility. Thus, increased levels of membrane DGLA per se is not toxic to germ cells, but these studies suggest that dietary fatty acids are processed differently than endogenous fatty acids to elicit a specific biological response (Watts and Browse 2006).

A role for polyunsaturated fatty acids for sperm attraction was recently uncovered in C. elegans. Amoeboid sperm crawl across the uterine wall to reach unfertilized 
oocytes. During oocyte formation, yolk lipoproteins synthesized in the intestinal tissue are trafficked to oocytes. These yolk particles contain polyunsaturated fatty acids that are precursors for signaling molecules that attract sperm. Sperm that fertilize fat-2 mutants, deficient in polyunsaturated fatty acid synthesis, show defects in sperm direction and velocity (Kubagawa et al. 2006). Genetic and biochemical analysis revealed that insulin/ IGF signaling pathways promote the formation of prostaglandins from polyunsaturated fatty acids, and these secreted prostaglandins attract sperm to the oocytes for fertilization (Edmonds et al. 2011).

\section{Involvement of fatty acid synthesis pathways in embryogenesis}

Lipid processing and synthesis genes are essential for establishing cell polarity in the C. elegans one-cell embryo. The first two committed steps in fatty acid synthesis, ACC and FAS, are encoded in C. elegans by the pod-2 and fasn-1 genes, respectively. Mutations in pod-2 and fasn-1 are maternal effect-lethal, and embryos from homozygous mutant mothers display osmotic sensitivity and loss of embryonic anterior-posterior polarity. After fertilization, the interaction between the male pronucleus centrosome and posterior cortex of the embryo is important for establishing anterior-posterior polarity. In pod-2 mutants, the male pronucleus centrosome fails to closely associate with the posterior cortex, and both this interaction and the cell polarity can be rescued by supplementing growth plates with various fatty acids (Rappleye et al. 2003). This suggests that fatty acid composition of membranes is regulated to maintain interactions with cytoskeletal elements such as centrosomes.

Cytochrome P450s are a class of lipid-modifying enzymes that regulates the production of various oxygenated lipid species. Mutations in two C. elegans cytochrome P450s, pod-7 and pod-8, and the cytochrome P450 reductase emb-8, which supplies electrons to cytochrome P450 enzymes, all have defects in establishing embryonic anterior-posterior polarity and osmotic defects. As lipid-modifying enzymes, POD-7 and POD-8 are proposed to act downstream from fatty acid synthesis genes, and as such, supplementing fatty acids was not able to rescue these embryonic defects (Benenati et al. 2009). While the biologically active products of POD-7/POD-8/ EMB-8 are still unknown, elucidating the identity of theses lipids will be critical for understanding how lipids establish embryonic polarity.

In addition to de novo fatty acid synthesis genes, the fatty acid modification genes are required for growth and development of C. elegans. Slow growth and embryonic lethality have been observed in elo-2 mutants, fat-2 mutants, and fat-3 mutants, all of which encode various desaturase and elongase components of the polyunsaturated fatty acid pathway (Watts and Browse 2002; Kniazeva et al. 2003; Watts et al. 2003). Furthermore, the fat-5;fat-6;fat-7 triple mutant strain, in which all stearoyl-CoA desaturase homologs are depleted, is not viable, revealing that the synthesis of unsaturated fatty acids is required for development, although the specific defects in embryogenesis have yet to be characterized (Brock et al. 2006).

The sterol regulatory element-binding protein (SREBP) transcription factors regulate genes involved in lipid metabolism from yeast to humans. The SREBP homologs in both C. elegans and Drosophila, sbp-1 and dSREBP, respectively, play important roles in regulating fatty acid and phospholipid biosynthesis and are required for viability. Supplementation of oleic acid partially rescues the larval lethality of Drosophila dSREBP mutants and reduced fertility and small size of $s b p-1$ (RNAi) worms, indicating the importance of unsaturated fatty acids in development (Kunte et al. 2006; Yang et al. 2006).

ACS genes convert long chain fatty acids to acyl-CoA esters, activating them for metabolic and signaling pathways. Recent work uncovered a new role for activated fatty acids in embryonic pattern formation. Drosophila $d A c s 1$ is ubiquitously expressed in early embryos, and loss of maternal and zygotic $d A c s 1$ activity causes fusion or deletion of abdominal segments. The expression domains of the gap genes $\mathrm{kni}$ and $\mathrm{Hb}$ are altered in $d A c s 1$ mutants, and consequently, the expression of the pair rule genes eve and $f t z$ is also disrupted (Zhang et al. 2011). However, it is unclear whether dAcs1 alters lipid composition of Drosophila embryos and, if it does, how this might mediate its gap gene function.

\section{Monomethyl branched chain fatty acids in C. elegans larval development}

A particular class of fatty acids, the monomethyl branched chain fatty acids, is present in a wide variety of organisms and is associated with disease states in humans, but little is known about its synthesis or function in eukaryotes. Monomethyl branched chain fatty acids are essential regulators of larval development in C. elegans (Kniazeva et al. 2004). RNAi of the elongase elo-5 significantly reduced the levels of the C15ISO and C17ISO monomethyl branched chain fatty acids and caused progeny to arrest in the L1 larval stage. The elo-5 larval arrest is robustly rescued by C17ISO dietary supplementation. This is not simply due to altered membrane fluidity, since elo-5 RNAi animals have a concomitant increase in the levels of $18: 1(n-7)$ fatty acids such that the saturation index is maintained (Kniazeva et al. 2004).

Other genes important for C17ISO function are acs-1 and the 3-keto-acyl-CoA reductase let-767 (Fig. 1). The LET-767 reductase is part of the elongation complex required to synthesize C17ISO from its C13ISO precursor (Entchev et al. 2008). ACS-1 is proposed to convert C17ISO precursors into activated acyl-CoA intermediates. Depleting either let-767 or acs-1 causes a decrease in C17ISO levels and larval arrest that can be rescued by supplementation with C17ISO (Kniazeva et al. 2008).

The C17ISO deprivation-induced larval arrest shows similarities to starvation-induced arrest. Both C17ISOand starvation-induced arrest are initiated within $5 \mathrm{~h}$ of hatching. Induction of larval arrest by starvation and by C17ISO depletion represses the cyclin-dependent kinase inhibitor CKI-1. However, unlike the starvation-induced 
larval arrest, C17ISO-induced arrest is independent of the DAF-2(IGF)/DAF-16(FOXO), suggesting that C17ISO levels mediate a larval arrest pathway that acts in parallel to DAF-2/DAF-16 (Kniazeva et al. 2008). Interestingly, the phospholipid translocase TAT-2 negatively regulates C17ISO-mediated growth regulation. Loss of tat-2 activity rescues the larval lethality of elo-5 mutants without restoring C17ISO synthesis. In C. elegans, C17ISO is incorporated into sphingolipids, but tat-2(lf) did not increase the relative amount of C17ISO in the sphingolipid fractions of elo-5 mutants. However, tat-2(lf) rescues the lethality of sptl-1 knockdown, a gene involved in sphingolipid synthesis. Thus, tat-2(lf) likely rescues C17ISO deprivation-induced arrest through a mechanism that alleviates the requirement of sphingolipids (Seamen et al. 2009).

A recent study implicates sphingolipid synthesis in the establishment of intestinal cell polarity and lumen formation during late stage embryogenesis and early larval development of C. elegans (Zhang et al. 2012). Iterative genetic screening of an intestinal polarity phenotype revealed a pathway for the synthesis of glycosphingolipids with a branched chain base and, most likely, a saturated fatty acid chain. Specifically, knockdown of the lipid synthesis genes pod-2, let-767, acs-1, and sptl-1 caused a loss of intestinal cell polarity and ectopic lateral lumen formation, and the supplementation of C17ISO rescued the acs1 intestinal polarity defect. Glycosphingolipid synthesis is required for polarity in growing larval intestinal cells, but not adult intestines, and the effects are reversible, suggesting that glycosphingolipids mediate the sorting of new components to expanding apical membrane domains (Zhang et al. 2012). Thus, incorporation of branched chain fatty acids into membrane lipids is not only important overall for viability, but also is specifically regulated to mediate cell polarity and tubulogenesis in C. elegans.

\section{The role of C17ISO and acs-1 in early development}

Depletion of elo-5 or acs-1 by RNAi leads to early larval arrest and lethality that can be rescued by including C17ISO in the diet (Kniazeva et al. 2004). However, the acs-1(RNAi) worms treated with C17ISO produced embryos that arrested in early development and failed to hatch. In the recent study from the Han laboratory (Kniazeva et al. 2012), the functions of C17ISO and acs-1 in early embryos were examined. The study revealed that embryos produced by the acs-1(RNAi) worms treated with C17ISO [hereafter referred to as acs-1(RNAi) ${ }^{C 17 I S O}$ ] displayed distinctive abnormalities during the first cell cycle. Normal embryos undergo vigorous membrane ruffling and pseudocleavage that are hallmarks of the contraction of the actomyosin network (Cowan and Hyman 2004), but embryos produced by acs-1(RNAi) ${ }^{\text {C17ISO }}$ worms displayed no such movement; instead, the cytoplasm appeared to occupy the entire volume of the egg. During cytokinesis of the first cell division, severe disruptions of membrane elongation occurred in early embryos produced by acs-1(RNAi) ${ }^{C 17 I S O}$ worms, resulting in membrane breaks, membrane branching, and failure to complete cytokinesis.

\section{ACS-1 acts in the somatic gonad to synthesize C17ISO phosphatidylethanolamine}

The depletion of acs-1 causes larval lethality; therefore, the examination of the maternal effect phenotypes necessitated a transgenic strain containing an extrachromosomal array carrying multiple copies of the wild-type acs-1 gene fused to green fluorescent protein (GFP). This array rescued the larval lethality in the acs-1 mutant. However, extrachromosomal arrays are inherently unstable, and some of these transgenic animals produced embryos with the acs-1(RNAi) ${ }^{C 17 I S O}$ phenotype. This was proof that endogenously produced C17ISO in combination with defective ACS-1 activity could also lead to defective embryogenesis and indicated that the phenotype arose when the rescuing array was lost in crucial tissues in which maternal activity of the acs-1 gene was necessary. The GFP fluorescence facilitated the analysis of mosaic animals to determine the tissues in which the array must be present to produce viable embryos. Strikingly, worms producing viable embryos always contained the array in their somatic gonad tissues, even when the array was lost in other tissues, such as in neurons. Likewise, all gonads that lost the array produced inviable embryos. The gonad expression was even more compelling because the expression of ACS-1::GFP in only one of the two gonad arms was able to rescue the embryonic-lethal phenotype only in the expressing gonad. This indicated that endogenous or dietary C17ISO can be toxic to developing embryos in the absence of ACS-1 activity and that ACS-1 acts in the somatic gonad to ensure that lipids of proper composition are provided maternally to developing oocytes. Interestingly, germline-specific RNAi of acs-1 did not cause embryonic lethality, indicating that ACS-1 functions solely in the somatic germline and is not required in the germ cells themselves.

The function of ACSs is to activate fatty acids for further synthetic or degradative reactions. To determine whether the depletion of acs-1 led to changes in lipid composition, Kniazeva et al. (2012) analyzed phospholipids in the acs-1(RNAi) ${ }^{C 17 I S O}$ worms. Tandem mass spectrometry and normal phase liquid chromatography analysis revealed that specific species of phosphatidylethanolamine containing C17ISO (C17ISO-PE) were depleted in the acs-1(RNAi) ${ }^{C 17 I S O}$ worms and that mysterious phospholipids containing C17ISO were produced instead (C17ISO-PL). This led to the conclusion that ACS-1 is required for the inclusion of C17ISO into phosphatidylethanolamine. When this did not occur because of the depletion of ACS-1, C17ISO became incorporated into an unknown phospholipid in the somatic gonad, which, when delivered to developing oocytes, leads to defects in membrane dynamics and cytokinesis.

\section{C17ISO-PE modulates inositol-3-phosphate $\left(\mathrm{IP}_{3}\right)$ signaling}

A clue as to how abnormal membrane lipids containing C17ISO may so drastically impact early embryonic events came from studying the genetic interactions between acs$1(R N A i)^{C 17 I S O}$ and genes regulating $\mathrm{IP}_{3}$ signaling. Phos- 


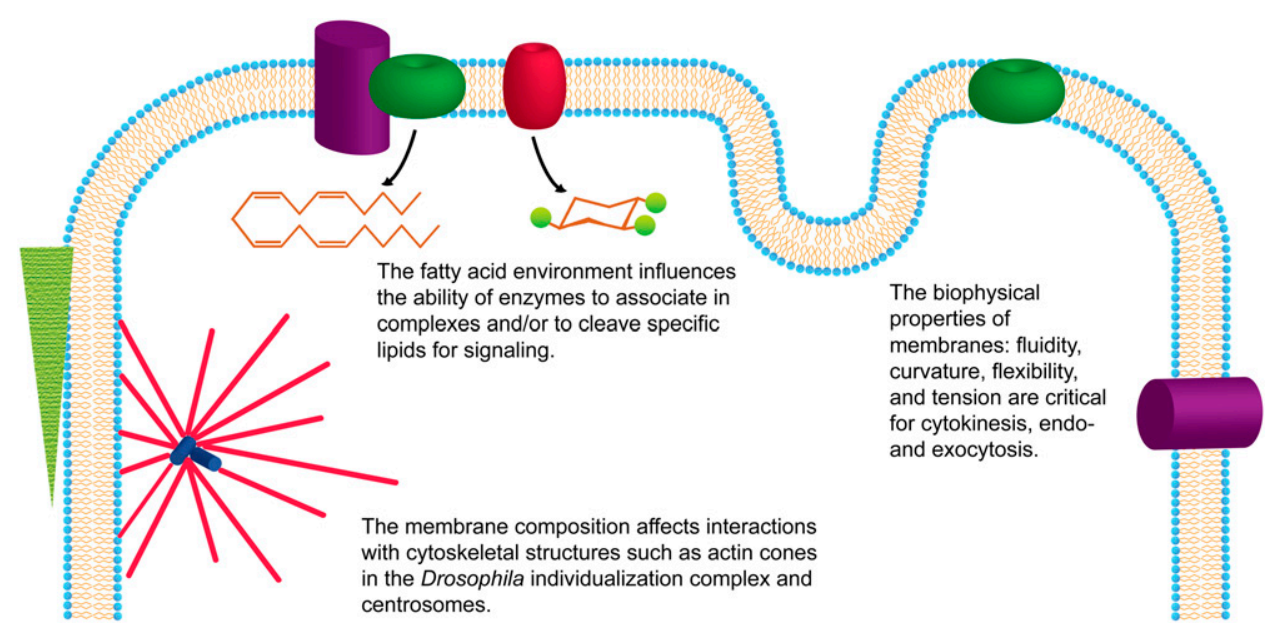

Figure 2. Schematic of the general mechanisms by which membrane composition may influence developmental processes. Disruptions of both dietary and de novo synthesis of fatty acids can alter the membrane composition, which in turn can affect interactions with cytoskeletal components, signaling, and the biophysical properties of membranes. Green, red, and purple cylinders represent integral membrane proteins such as phospholipases. The green triangle represents an actin cone.

pholipase C cleaves the membrane phosphatidylinositol to produce soluble $\mathrm{IP}_{3}$. Receptors in the endoplasmic reticulum respond to $\mathrm{IP}_{3}$ by releasing calcium ions, which further regulate many developmental processes, including fertilization, proliferation, membrane contractions, and vesicle secretions (Berridge 2009). C. elegans contains a single $\mathrm{IP}_{3}$ receptor, ITR-1. Embryos carrying loss-offunction mutations in the itr-1 gene displayed an egg-filling multinucleate phenotype similar to the acs-1(RNAi) ${ }^{\text {C17ISO }}$ embryos (Walker et al. 2002). This led Kniazeva et al. (2012) to treat itr-1 gain-of-function mutants as well as ipp-5 lossof-function mutants, both of which have hyperactive $\mathrm{IP}_{3}$ signaling, with acs-1(RNAi) in the presence or absence of C17ISO. They found that the embryonic abnormalities were suppressed in both of the hyperactive signaling mutants, indicating that $\mathrm{IP}_{3}$ signaling may occur less efficiently in the C17ISO-PL membrane environment than it does in the C17ISO-PE membrane environment. Thus, proper membrane composition appears to be critical for important signaling events during early development.

\section{Conclusions}

The biophysical properties of membranes are under tight regulation by multiple lipid synthesis genes. The fatty acid environment is known to change membrane properties, affecting membrane fluidity, tension, curvature, and formation of membrane microdomains. These membrane properties can affect important cellular processes, such as cytokinesis, exocytosis, endocytosis, and the accessibility of lipid-modifying enzymes to their substrates (Fig. 2). How these relate to more complex interactions of cells is less well-understood, Thus, we are only just beginning to understand how the lipid composition of membranes and specific fatty acids affect animal development.

Emerging studies in this area have revealed a role for de novo fatty acid synthesis in embryos, which likely involves the generation of a membrane environment condu- cive to optimal interactions among cytoskeletal components. The new study by Kniazeva et al. (2012) suggests another mechanism of action-that signaling molecules, such as $\mathrm{IP}_{3}$, may not be generated appropriately when the membrane environment is not optimal. Future genetic and biochemical studies, combined with advances in lipidomic analysis, will not only allow for the identification of new lipid species, but continue to reveal novel vital functions for these lipids in cell biology and development.

\section{Acknowledgments}

We thank Dr. Wendy Hanna-Rose for helpful comments on the manuscript. Research in the Watts laboratory is funded by the National Institutes of Health (USA) R01-DK074114.

\section{References}

Abu-Elheiga L, Matzuk MM, Kordari P, Oh W, Shaikenov T, Gu Z, Wakil SJ. 2005. Mutant mice lacking acetyl-CoA carboxylase 1 are embryonically lethal. Proc Natl Acad Sci 102: 12011-12016.

Baker KD, Thummel CS. 2007. Diabetic larvae and obese flies-emerging studies of metabolism in Drosophila. Cell Metab 6: 257-266.

Benenati G, Penkov S, Muller-Reichert T, Entchev EV, Kurzchalia TV. 2009. Two cytochrome P450s in Caenorhabditis elegans are essential for the organization of eggshell, correct execution of meiosis and the polarization of embryo. Mech Dev 126: 382-393.

Berridge MJ. 2009. Inositol trisphosphate and calcium signalling mechanisms. Biochim Biophys Acta 1793: 933-940.

Brock TJ, Browse J, Watts JL. 2006. Genetic regulation of unsaturated fatty acid composition in C. elegans. PLoS Genet 2: e108. doi: 10.1371/journal.pgen.0020108.

Chirala SS, Chang H, Matzuk M, Abu-Elheiga L, Mao J, Mahon K, Finegold M, Wakil SJ. 2003. Fatty acid synthesis is essential in embryonic development: Fatty acid synthase null mutants and most of the heterozygotes die in utero. Proc Natl Acad Sci 100: 6358-6363. 
Cowan CR, Hyman AA. 2004. Asymmetric cell division in C. elegans: Cortical polarity and spindle positioning. Annu Rev Cell Dev Biol 20: 427-453.

Edmonds JW, Prasain JK, Dorand D, Yang Y, Hoang HD, Vibbert J, Kubagawa HM, Miller MA. 2011. Insulin/FOXO signaling regulates ovarian prostaglandins critical for reproduction. Dev Cell 19: 858-871.

Ellis JM, Frahm JL, Li LO, Coleman RA. 2010. Acyl-coenzyme A synthetases in metabolic control. Curr Opin Lipidol 21: 212217.

Entchev EV, Schwudke D, Zagoriy V, Matyash V, Bogdanova A, Habermann B, Zhu L, Shevchenko A, Kurzchalia TV. 2008. LET-767 is required for the production of branched chain and long chain fatty acids in Caenorhabditis elegans. J Biol Chem 283: $17550-17560$.

Fahy E, Cotter D, Sud M, Subramaniam S. 2011. Lipid classification, structures and tools. Biochim Biophys Acta 1811: 637-647.

Guillou H, Zadravec D, Martin PG, Jacobsson A. 2010. The key roles of elongases and desaturases in mammalian fatty acid metabolism: Insights from transgenic mice. Prog Lipid Res 49: $186-199$.

Haeggstrom JZ, Funk CD. 2011. Lipoxygenase and leukotriene pathways: Biochemistry, biology, and roles in disease. Chem Rev 111: 5866-5898.

Jung A, Hollmann M, Schafer MA. 2007. The fatty acid elongase NOA is necessary for viability and has a somatic role in Drosophila sperm development. J Cell Sci 120: 29242934.

Kniazeva M, Sieber M, McCauley S, Zhang K, Watts JL, Han M. 2003. Suppression of the ELO-2 FA elongation activity results in alterations of the fatty acid composition and multiple physiological defects, including abnormal ultradian rhythms, in Caenorhabditis elegans. Genetics 163: 159-169.

Kniazeva M, Crawford QT, Seiber M, Wang CY, Han M. 2004. Monomethyl branched-chain fatty acids play an essential role in Caenorhabditis elegans development. PLoS Biol 2: E257. doi: 10.1371/journal.pbio.0020257.

Kniazeva M, Euler T, Han M. 2008. A branched-chain fatty acid is involved in post-embryonic growth control in parallel to the insulin receptor pathway and its biosynthesis is feedback-regulated in C. elegans. Genes Dev 22: 2102-2110.

Kniazeva M, Shen H, Euler T, Wang C, Han M. 2012. Regulation of maternal phospholipid composition and $\mathrm{IP}_{3}$-dependent embryonic membrane dynamics by a specific fatty acid metabolic event in C. elegans. Genes Dev 26: 554-566.

Kubagawa HM, Watts JL, Corrigan C, Edmonds JW, Sztul E, Browse J, Miller MA. 2006. Oocyte signals derived from polyunsaturated fatty acids control sperm recruitment in vivo. Nat Cell Biol 8: 1143-1148.

Kunte AS, Matthews KA, Rawson RB. 2006. Fatty acid auxotrophy in Drosophila larvae lacking SREBP. Cell Metab 3: 439-448.

Rappleye CA, Tagawa A, Le Bot N, Ahringer J, Aroian RV. 2003. Involvement of fatty acid pathways and cortical interaction of the pronuclear complex in Caenorhabditis elegans embryonic polarity. BMC Dev Biol 3: 8. doi: 10.1186/1471213X-3-8.

Sakamoto T, Inoue T, Otomo Y, Yokomori N, Ohno M, Arai H, Nakagawa Y. 2012. Deficiency of cardiolipin synthase causes abnormal mitochondrial function and morphology in germ cells of Caenorhabditis elegans. I Biol Chem 287: 45904601.

Seamen E, Blanchette JM, Han M. 2009. P-type ATPase TAT-2 negatively regulates monomethyl branched-chain fatty acid mediated function in post-embryonic growth and develop- ment in C. elegans. PLoS Genet 5: e1000589. doi: 10.1371/ journal.pgen.1000589.

Shevchenko A, Simons K. 2010. Lipidomics: Coming to grips with lipid diversity. Nat Rev Mol Cell Biol 11: 593-598.

Simons K, Sampaio JL. 2011. Membrane organization and lipid rafts. Cold Spring Harb Perspect Biol 3: a004697. doi: 10.1101/cshperspect.a004697.

Smith WL, Urade Y, Jakobsson P-J. 2011. Enzymes of the cyclooxygenase pathways of prostanoid biosynthesis. Chem Rev 111: 5821-5865.

Steinhauer J, Gijon MA, Riekhof WR, Voelker DR, Murphy RC, Treisman JE. 2009. Drosophila lysophospholipid acyltransferases are specifically required for germ cell development. Mol Biol Cell 20: 5224-5235.

Szafer-Glusman E, Giansanti MG, Nishihama R, Bolival B, Pringle J, Gatti M, Fuller MT. 2008. A role for very-longchain fatty acids in furrow ingression during cytokinesis in Drosophila spermatocytes. Curr Biol 18: 1426-1431.

van Meer G, Voelker DR, Feigenson GW. 2008. Membrane lipids: Where they are and how they behave. Nat Rev Mol Cell Biol 9: 112-124.

Walker DS, Gower NJD, Ly S, Bradley GL, Baylis HA. 2002. Regulated disruption of inositol 1,4,5-trisphosphate signaling in Caenorhabditis elegans reveals new functions in feeding and embryogenesis. Mol Biol Cell 13: 1329-1337.

Wallis JG, Watts JL, Browse J. 2002. Polyunsaturated fatty acid synthesis: What will they think of next? Trends Biochem Sci 27: 467-473.

Watts JL. 2009. Fat synthesis and adiposity regulation in Caenorhabditis elegans. Trends Endocrinol Metab 20: 58-65.

Watts JL, Browse J. 2002. Genetic dissection of polyunsaturated fatty acid synthesis in Caenorhabditis elegans. Proc Natl Acad Sci 99: 5854-5859.

Watts JL, Browse J. 2006. Dietary manipulation implicates lipid signaling in the regulation of germ cell maintenance in C. elegans. Dev Biol 292: 381-392.

Watts JL, Phillips E, Griffing KR, Browse J. 2003. Deficiencies in C20 polyunsaturated fatty acids cause behavioral and developmental defects in Caenorhabditis elegans fat-3 mutants. Genetics 163: 581-589.

Yang F, Vought BW, Satterlee JS, Walker AK, Jim Sun ZY, Watts JL, DeBeaumont R, Saito RM, Hyberts SG, Yang S, et al. 2006. An ARC/Mediator subunit required for SREBP control of cholesterol and lipid homeostasis. Nature 442: 700-704.

Zhang Y, Zhang Y, Gao Y, Zhao X, Wang Z. 2011. Drosophila long-chain acyl-CoA synthetase acts like a gap gene in embryonic segmentation. Dev Biol 353: 259-265.

Zhang H, Abraham N, Khan LA, Hall DH, Fleming JT, Gobel V. 2012. Apicobasal domain identities of expanding tubular membranes depend on glycosphingolipid biosynthesis. Nat Cell Biol 13: 1189-1201. 


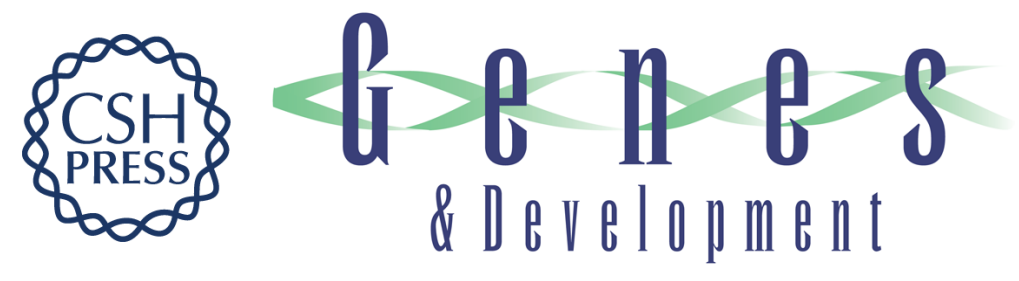

\section{Emerging roles for specific fatty acids in developmental processes}

Tracy L. Vrablik and Jennifer L. Watts

Genes Dev. 2012, 26:

Access the most recent version at doi:10.1101/gad.190777.112

Related Content Regulation of maternal phospholipid composition and IP3-dependent embryonic membrane dynamics by a specific fatty acid metabolic event in C. elegans Marina Kniazeva, Huali Shen, Tetyana Euler, et al.

Genes Dev. March , 2012 26: 554-566

References This article cites 38 articles, 11 of which can be accessed free at: http://genesdev.cshlp.org/content/26/7/631.full.html\#ref-list-1

Articles cited in:

http://genesdev.cshlp.org/content/26/7/631.full.html\#related-urls

\section{License}

Email Alerting

Receive free email alerts when new articles cite this article - sign up in the box at the top

Service right corner of the article or click here.

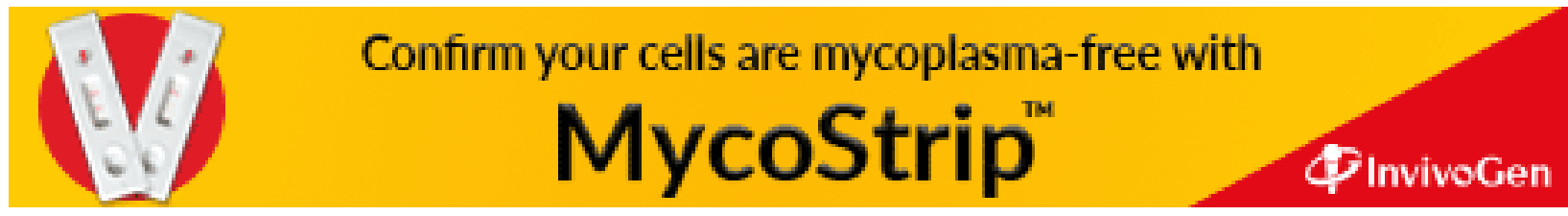

